\title{
At-home training with closed-loop augmented-reality cueing device for improving gait in patients with Parkinson disease
}

\author{
Alberto J. Espay, MD, MSc; ${ }^{1 *}$ Yoram Baram, PhD; ${ }^{2}$ Alok Kumar Dwivedi, PhD; ${ }^{3}$ Rakesh Shukla, PhD; ${ }^{3}$ \\ Maureen Gartner, RN, MEd; ${ }^{1}$ Laura Gaines, BA, CCRC; ${ }^{1}$ Andrew P. Duker, MD; ${ }^{1}$ Fredy J. Revilla, MD $^{\mathbf{1}}$ \\ ${ }^{1}$ The Neuroscience Institute, Department of Neurology, Movement Disorders Center, University of Cincinnati, Cincin- \\ nati, $\mathrm{OH} ;{ }^{2}$ Department of Computer Science, Technion-Israel Institute of Technology, Haifa, Israel; ${ }^{3}$ Center for Bio- \\ statistical Services, Department of Environmental Health, University of Cincinnati, Cincinnati, OH
}

\begin{abstract}
Shuffling and freezing while walking can impair function in patients with Parkinson disease (PD). Open-loop devices that provide fixed-velocity visual or auditory cues can improve gait but may be unreliable or exacerbate freezing of gait in some patients. We examined the efficacy of a closedloop, accelerometer-driven, wearable, visual-auditory cueing device in 13 patients with PD with off-state gait impairment at baseline and after 2 weeks of twice daily (30 minute duration) at-home use. We measured gait velocity, stride length, and cadence using a validated electronic gait-analysis system. Subjects underwent standard motor assessment and completed a self-administered Freezing of Gait Questionnaire (FOGQ) (range 0-24; lower is better). After training, device use enhanced walking velocity $(61.6 \pm 20.1 \mathrm{~cm} / \mathrm{s}$ to $72.6 \pm$ $26.5 \mathrm{~cm} / \mathrm{s}, p=0.006)$ and stride length $(74.3 \pm 16.4 \mathrm{~cm}$ to $84.0 \pm$ $18.5 \mathrm{~cm}, p=0.004)$. Upon device removal, walking velocity $(64.5 \pm 21.4 \mathrm{~cm} / \mathrm{s}$ to $75.4 \pm 21.5 \mathrm{~cm} / \mathrm{s}, p<0.001)$ and stride length $(79.0 \pm 20.3 \mathrm{~cm}$ to $88.8 \pm 17.7 \mathrm{~cm}, p=0.003)$ exhibited a greater magnitude of change, suggesting immediate residual benefits. Also upon device removal, nearly 70 percent of subjects improved by at least 20 percent in either walking velocity, stride length, or both. An overall improvement in gait was measured by the FOGQ (14.2 \pm 1.9 to $12.4 \pm 2.5, p=0.02)$. Although issues related to compliance and response variability render a definitive interpretation of study outcome difficult, devices using closed-loop sensory feedback appear to be effective and desirable nonpharmacologic interventions to improve walking in selected individuals with PD.
\end{abstract}

Key words: cueing device, festination, freezing of gait, gait, gait impairment, Parkinson disease, PD, rehabilitation, virtual reality device, visual feedback.

\section{INTRODUCTION}

Gait impairment in the form of shuffling, short steps, or freezing often occurs during the course of Parkinson disease (PD), generating substantial disability [1]. It is well recognized that auditory, visual, or tactile stimuli can improve gait in patients with PD [2]. As a result, devices that exploit the sensory cueing-related modification of gait in patients with PD have been proposed with limited success for ongoing use [3-4]. These devices employ open-loop strategies; that is, they impose on the patient a sensory signal, generated by an external source,

\footnotetext{
Abbreviations: FOGQ = Freezing of Gait Questionnaire, $\mathrm{PD}=$ Parkinson disease, UPDRS = Unified Parkinson Disease Rating Scale.

*Address all correspondence to Alberto J. Espay, MD, MSc; Department of Neurology, University of Cincinnati, 260 Stetson St, Suite 3200, Cincinnati, OH 45267-0525; 513558-4035; fax: 513-558-7015.

Email: alberto.espay@uc.edu

DOI:10.1682/JRRD.2009.10.0165
} 
that is not affected by the patient's own motion. Examples are fixed-velocity visual cues [5] or rhythmic auditory cues [6]. While open-loop stimulation may offer some benefit to some patients [3,7], such cues were found to cause confusion and exacerbate freezing of gait in others [3]. Indeed, it is well known that the stabilization of open-loop dynamic systems, which are inherently unstable, can only be achieved by feedback, that is closed-loop, systems [8]. The key role played by sensory feedback in gait stabilization and control, i.e., where the feedback signal is generated by the patient's own motion, has been demonstrated by the gait improvements in patients with PD when they are provided with visual markings on the ground [9-10]. At the same time, a clinical study addressing the role of visual feedback in maintaining postural balance in stance demonstrated that feedback increases postural stability in standing tasks, indicating a more prominent role for closed-loop (feedback) control over open-loop (feed-forward) control [11].

The development of a wearable virtual reality device driven by inertial sensors that delivers earth-stationary visual feedback cues has provided an opportunity to assess a closed-loop system of sensory (visual-auditory) feedback stimuli to aid dysfunctional gait in patients with PD. We sought to examine the effect of a wearable, closed-loop, visual-auditory cueing system on parkinsonian gait in the "off" state, when the magnitude of gait impairment is expected to be largest. Preliminary clinical studies [3,12] have demonstrated that the closed-loop feedback device significantly improves gait speed while in use and appears to induce a residual benefit for several days by dynamically delivering a tiled floor pattern adapted to the patient's own motion. It is believed that the optical flow provides a reassuring feedback effect, informing the patient of motion being performed and matching visual feedback from foot-tile matching to auditory feedback from clicking obtained for each step. These elements help produce a balanced rhythmic walk. In contrast to the open-loop strategies, this device also appears to produce and maintain a high degree of patient confidence, comfort, and safety [3]. To the best of our knowledge, this is the first study assessing the feasibility and effects of at-home training with a closed-loop sensoryfeedback device in patients with PD.

\section{METHODS}

\section{Subjects}

We recruited consecutive, consenting patients with PD who had off-state gait shuffling, festination, and/or freezing and a score $>1$ on the Unified Parkinson Disease Rating Scale (UPDRS) III item 29 and who did not require continuous ambulatory aid from the Movement Disorders Center at the University of Cincinnati. Patients spent $>3$ hours/day in the off state. We did not study patients with primary on-state freezing to maximize homogeneity of the target population. Patients had been on a stable dose of antiparkinsonian medications and required no medication adjustments during the course of the study. The following exclusion criteria were used: (1) prior functional neurosurgery; (2) musculoskeletal disorders, such as severe arthritis, knee surgery, hip surgery, or any other condition that the investigators determined would impair assessment of gait; (3) history of stroke; (4) cerebellar, vestibular, or sensory ataxia; and (5) Mini-Mental State Examination score <24.

\section{Visual-Auditory Walker}

The device is composed of a small measurementcomputation unit attached to the patient's clothing, a head-mounted microdisplay, and earphones (Figure 1). The measurement-computation unit is composed of a

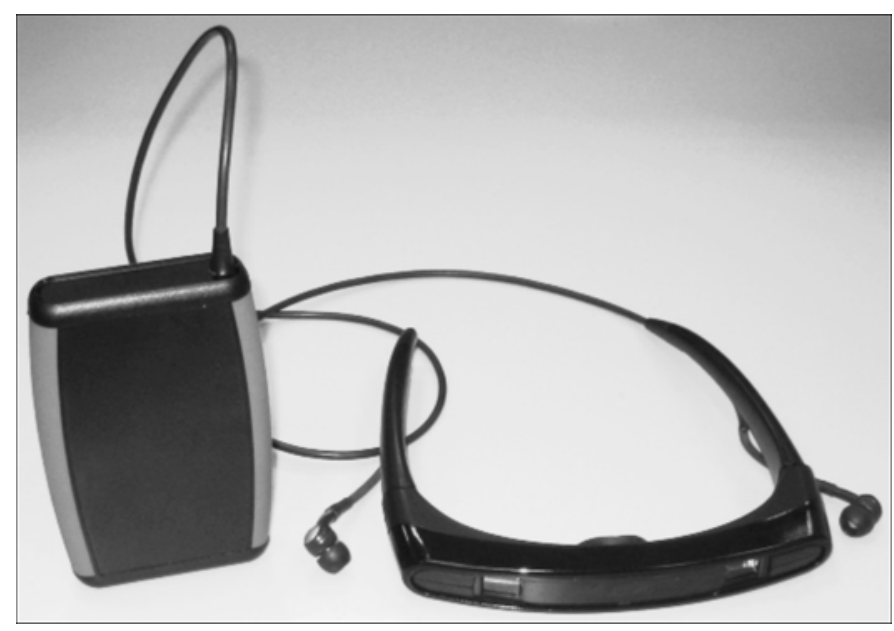

Figure 1.

Virtual (augmented) reality goggles used in this study containing built-in LCD screen, which projects floor tiles when subjects are moving, and earphones that sound step-matched cue as determined by connected sensor strapped at belt. 
multiaxial accelerometer, a compass, and a microcontroller. The apparatus, operating in an adaptive closed-loop mode, displays a life-size virtual checkerboard-tiled floor superimposed on the real world with specialized seethrough glasses. The closed-loop (or feedback) concept (as opposed to open-loop) implies that the speed of the cues is not externally set but, rather, is an outcome (and indeed a duplicate) of the walking speed of the user. The visual effect is the same as that created by walking over earth-stationary cues (i.e., a real tiled floor). Similarly, the rhythm of the auditory cue is determined by the rhythm of the steps, not vice versa. The user then regulates the gait pattern to create a constant optical flow and a rhythmic auditory cue. The virtual (augmented) floor responds dynamically to the patient's own motion and "moves" toward him or her at the speed set by the user as measured by the accelerometer. The tiled floor acts as a moving visual display whose speed is generated in a natural feedback fashion by the patient's own motion, much like earth-stationary visual cues. The grid allows for patients to step on the tiles with long strides as they walk, though they do not enlarge or modify based on previous step length. A steady gait synchronizes the patient's own steps with the virtual tiles and the auditory cues, thus "rewarding" the patient for making the effort. Subjects can view real-world obstacles without difficulty. Subjects with corrective lenses are able to use the device without difficulty. Additional auditory feedback from the patient's own steps is provided through earphones. Subjects hear the auditory cue produced by the steps through the earphones. The auditory feedback is continuous so long as patients are walking steadily, producing a rhythm they hear based on their gait pattern.

\section{Gait System}

The validated gait analysis system (GAITRite, CIR Systems, Inc; Havertown, Pennsylvania) used in this study consists of a 4-meter electronic walkway that contains six sensor pads encapsulated in a roll-up carpet to produce an active area 24 inches $(61 \mathrm{~cm})$ wide and 144 inches $(366 \mathrm{~cm})$ long [13]. In this arrangement, the active area is a grid (48 sensors by 288 sensors placed on 0.5 inch $[1.27 \mathrm{~cm}]$ centers) totaling 13,824 sensors. The walkway is portable, can be laid over any flat surface, requires minimal setup and test time, and requires no placement of any devices on the patient. The application software controls the functionality of the walkway, processes the raw data into footfall patterns, and computes temporal (timing) and spatial (distance) gait parameters, including stride length, cadence, and velocity.

\section{Study Evaluations}

Subjects were asked to walk at their usual pace on a defined 10-meter path encompassing the GAITRite walkway beginning and ending at the same point under five conditions: (1) no sensory feedback (no device, baseline); (2) visual-auditory device positioned but deactivated (device off), (3) visual-auditory device positioned with visual-only feedback activated, (4) visual and auditory feedback activated, and (5) again with no sensory feedback (device off, immediate residual effect). Each condition was repeated three times and the average of these was used for analysis. Due to the nature of the intervention, the order of the assessments was not randomized and subject blinding was unattainable (signal noise in device would have worsened gait in any control group) but comparison of conditions 1 (no device) and 2 (device off) served to determine whether a placebo-like response could be elicited when the device was not yet providing visual-auditory feedback. Subjects were assessed at two time points: baseline (visit 1) and after 2 weeks of athome use (visit 2). In between these in-clinic evaluations, subjects were instructed to use the visual-auditory device while walking for no less than two 30-minute periods a day, regardless of their medication state. We emphasized use during periods of expected off-related gait worsening. Subjects were not restricted in their choice of walking inside or outside and were encouraged to incorporate training with their regular activities. Since subjects were recruited on the basis of their off-related gait impairment, all study assessments took place during the "practically defined off period," that is, in the morning at least 12 hours since the last dose of any antiparkinsonian medication. We administered a standard motor examination with the UPDRS III [14] and asked subjects to complete the self-administered Freezing of Gait Questionnaire (FOGQ, range 0-24; lower is better) [15] to ascertain their motor and gait-specific function.

\section{Data Analysis}

We used the primary outcome measures (gait velocity, stride length, and cadence) to assess the effect of the device under various conditions. To accomplish this, we evaluated the effects of the various conditions (no device, device without feedback, visual-only feedback, visualauditory feedback, and no device for immediate residual) on each outcome variables using repeated measures analysis 
of variance followed by post hoc multiple comparisons with Bonferroni adjustment for each visit separately. The multiple comparisons were for each of the conditions versus baseline condition (condition 1). We examined the effect of training on each outcome using paired $t$-tests for each condition separately. Secondary outcome measures included changes in total FOGQ score, UPDRS III score, axial UPDRS III score, and freezing frequency between baseline and follow-up. We calculated the axial UPDRS III subscore by adding motor items $18-19$ and $27-30$ as described in a previous study [16], to determine whether in addition to gait, other axial motor features of PD were affected by the device. We used paired $t$-tests to compare the secondary outcomes between the visits. As an exploratory analysis, we used multiple regression analysis to assess the effect of baseline UPDRS on the absolute change in velocity and stride length between condition 5 at baseline and follow-up, adjusting for disease duration. The significance criterion for the regression analysis was kept at 0.05 . We considered $p$-values less than 5 percent significant.

\section{RESULTS}

\section{Clinical Descriptions}

Fifteen patients were enrolled, but two did not feel comfortable using the glasses (because they were "clunky" or "embarrassing to use in public") and did not train at home as instructed. The patients (6 male, $73.3 \pm$ 11.7 years old, disease duration $12.1 \pm 4.2$ years, levodopa equivalents $1,413.8 \pm 786.2 \mathrm{mg}$ ) had a UPDRS III score (off-state during both assessments) of $28.1 \pm$ 11.1 at the first assessment and $24.1 \pm 9.1$ at the second assessment (all data presented as mean \pm standard deviation unless otherwise noted). Patients reported varying preference of sensory feedback: four subjects favored the combination of auditory and visual feedback, three subjects preferred auditory stimulation alone, three visual feedback alone, and two neither (data missing for one subject). The gait parameters obtained for each condition with the gait analysis system are shown in the Table.

Table.

Gait parameters per condition (data presented as mean \pm standard deviation).

\begin{tabular}{|c|c|c|c|}
\hline Condition & Velocity $(\mathrm{cm} / \mathrm{s})$ & Stride Length (cm) & Cadence (steps/min) \\
\hline \multicolumn{4}{|l|}{ No Device (baseline) } \\
\hline Visit 1 & $64.2 \pm 18.8$ & $75.0 \pm 15.4$ & $104.4 \pm 22.0$ \\
\hline Visit 2 & $69.2 \pm 22.8$ & $81.1 \pm 19.9$ & $108.9 \pm 23.3$ \\
\hline$\% \Delta$ & $7.7 \pm 14.8$ & $8.1 \pm 15.1$ & $7.1 \pm 23.0$ \\
\hline \multicolumn{4}{|c|}{ Device Without Feedback (off) } \\
\hline Visit 1 & $60.6 \pm 18.3$ & $73.9 \pm 15.9$ & $117.1 \pm 27.3$ \\
\hline$\% \Delta$ & $18.7 \pm 23.2$ & $16.6 \pm 19.4$ & $-3.7 \pm 15.6$ \\
\hline \multicolumn{4}{|c|}{ Visual-Only Feedback } \\
\hline Visit 1 & $56.4 \pm 22.0$ & $69.6 \pm 20.5$ & $105.3 \pm 26.2$ \\
\hline Visit 2 & $69.5 \pm 26.8$ & $80.4 \pm 22.6$ & $109.2 \pm 24.5$ \\
\hline$\% \Delta$ & $26.5 \pm 29.1^{*}$ & $19.2 \pm 28.4$ & $5.9 \pm 20.7$ \\
\hline$\% \Delta$ & $17.1 \pm 20.4^{\dagger}$ & $13.6 \pm 12.7^{\dagger}$ & $3.3 \pm 12.6$ \\
\hline \multicolumn{4}{|c|}{ No Device (immediate residual) } \\
\hline Visit 1 & $64.5 \pm 21.4$ & $79.0 \pm 20.3$ & $107.4 \pm 42.3$ \\
\hline Visit 2 & $75.4 \pm 21.5$ & $88.8 \pm 17.7$ & $114.6 \pm 25.8$ \\
\hline$\% \Delta$ & $18.7 \pm 14.4^{\ddagger}$ & $14.9 \pm 15.2^{\ddagger}$ & $14.2 \pm 35.0$ \\
\hline \multicolumn{4}{|c|}{$\begin{array}{l}\text { *Significant difference between visits } p<0.05 \text {. } \\
{ }^{\dagger} \text { Significant difference between visits } p<0.01 \text {. } \\
{ }^{\dagger} \text { Significant difference between visits } p<0.001 \text {. } \\
\text { Diff = Visit } 2-\text { Visit } 1, \% \Delta=\text { percent change ([Diff/Visit 2] } \times 100 \text { ). }\end{array}$} \\
\hline
\end{tabular}




\section{Condition Effect by Visit}

At visit 1 , a significant effect of device on gait velocity was noted under various conditions $(p=0.006)$. The multiple pairwise comparisons revealed that condition 2 was not significantly different from condition 1 ( $p=$ 0.39). However, condition 3 was significantly different from condition $1(p=0.008)$. At visit 2 , the effect of device on gait velocity for various conditions was found to be statistically significant $(p=0.03)$. In post hoc pairwise comparisons, condition 5 was significantly different from condition 1 ( $p=0.01)$, showing that a residual effect of device on gait velocity existed. No significant changes in mean stride length were noted at different conditions on visit $1(p=0.18)$. However, a significant improvement in stride length was found at visit 2. Only condition 5 was found to be significant as compared with condition $1(p=0.008)$. Cadence was not significantly different under any condition for any visit. In summary, a condition effect on gait velocity as well as stride was found. The positive effects of device on gait velocity and stride were mainly observed at the second visit between condition 5 and condition 1 .

\section{Training Effect for Condition 1}

A trend for improvement in velocity $(64.2 \pm 18.8 \mathrm{~cm} / \mathrm{s}$ to $69.2 \pm 22.8 \mathrm{~cm} / \mathrm{s}, p=0.05)$ and stride length $(75.0 \pm$ $15.4 \mathrm{~cm}$ to $81.1 \pm 19.9 \mathrm{~cm}, p=0.08)$ was noted. Changes in cadence $(104.4 \pm 22.0$ steps/min to $108.9 \pm 23.3$ steps/ min) were not significant. Gait velocity improved by at least 10 percent in six subjects and by more than 20 percent in two. Stride length showed similarly graded responses (Figure 2).

\section{Training Effect for Condition 2}

A significant effect was noted of training on gait velocity $(p=0.02)$ and stride $(p=0.02)$ but not on cadence $(p=0.70)$ for condition 2 . This result indicates that improved training effects were obtained with the use of the device.

\section{Training Effect for Condition 3}

Under condition 3, significant improvement in gait velocity was noted $(p=0.03)$. Also, a trend toward improvement in stride length was found $(p=0.06)$. However, again no change was noted in cadence for condition 3 $(p=0.51)$.

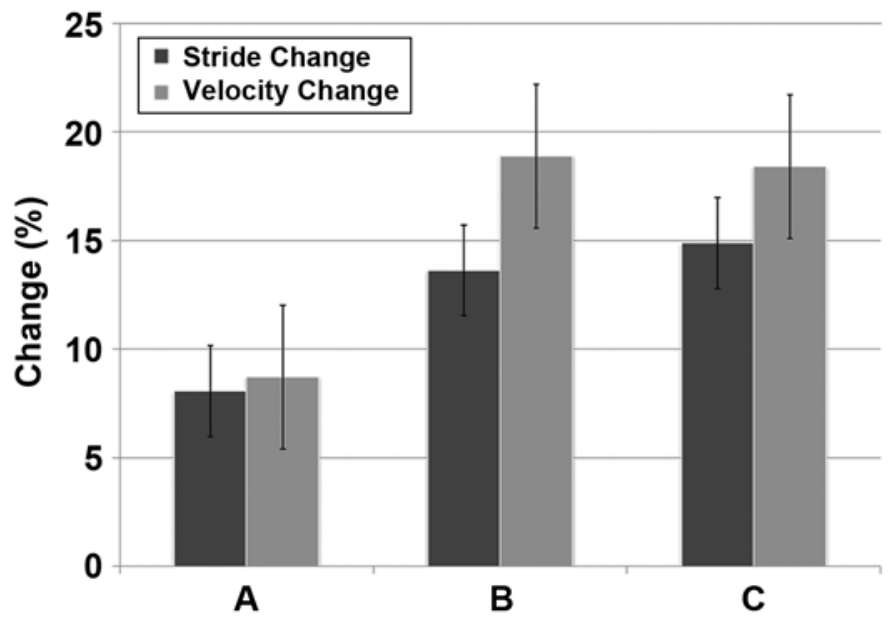

Figure 2.

Percent change of velocity and stride length due to training effect ( $\mathrm{A}=$ condition 1, day 1 vs 14, no sensory feedback), combined device and training effects ( $\mathrm{B}=$ condition 4 , day 1 vs 14 , sensory feedback), and combined training and immediate residual effects $(\mathrm{C}=$ condition 5 , day 1 vs 14, no sensory feedback). Vertical bars denote standard error of the mean.

\section{Training Effect for Condition 4}

An improvement was found in gait velocity (61.6 \pm $20.1 \mathrm{~cm} / \mathrm{s}$ to $72.6 \pm 26.5 \mathrm{~cm} / \mathrm{s}, p=0.006)$ and stride length $(74.3 \pm 16.4 \mathrm{~cm}$ to $84.0 \pm 18.5 \mathrm{~cm} ; p=0.004)$. The improvement in cadence was not significant. Gait velocity improved by at least 20 percent in eight subjects and by more than 30 percent in five. Stride length improved by at least 10 percent in nine subjects and by more than 20 percent in five (Figure 2).

\section{Training Effect at Condition 5}

Marked improvement was found in gait velocity $(64.5 \pm 21.4 \mathrm{~cm} / \mathrm{s}$ to $75.4 \pm 21.5 \mathrm{~cm} / \mathrm{s}, p<0.001)$ and stride length $(79.0 \pm 20.3 \mathrm{~cm}$ to $88.8 \pm 17.7 \mathrm{~cm} ; p=$ 0.003 ), but not cadence. Gait velocity improved by at least 10 percent in nine subjects and by more than 30 percent in three. Stride length improved by more than 10 percent in nine subjects and by more than 20 percent in five (Figure 2).

\section{Training Effect Summary}

The results indicate that the training effect became more magnified under condition 4 and condition 5 for gait velocity as well as stride compared with condition 1 . Also, a significant improvement in velocity and stride 
was found for condition 5 compared with condition 1 , especially at visit 2 . Thus, an effect of training and condition on velocity and stride exists.

\section{Unified Parkinson Disease Rating Scale and Freezing of Gait Questionnaire}

Higher baseline UPDRS scores predicted residual velocity and stride length improvement $(p=0.002)$ adjusted for disease duration, indicating that patients with more advanced disease showed greater benefit. The FOGQ improved from $14.2 \pm 1.9$ to $12.4 \pm 2.5(p=0.02)$. A trend toward decreasing frequency of freezing was noted from visit 1 (2.92 episodes/day) to visit 2 (2.54 episodes/day, $p=0.09$ ). The UPDRS III at visit 2 was $24.1 \pm$ 9.1 , lower than at visit $1(28.1 \pm 11.1)$ despite no changes in medications between the two off-state assessments $(p=$ 0.009). A trend toward a reduction in the axial UPDRS scores was found at visit $2(p=0.07)$.

\section{DISCUSSION}

Training with augmented reality visual-auditory feedback cues improved gait velocity by 18 to 20 percent in the immediate carryover state in a cohort of patients with PD who had substantial off-state gait impairment following a 2-week at-home period of self-training. Contrary to our expectations, the benefits were not observed within each visit, immediately upon device activation, or at the initial posttraining evaluation before device activation, but as a robust immediate residual or carryover effect at the end of the last assessment. In fact, nearly 70 percent of the subjects showed residual improvement of at least 20 percent in velocity, stride length, or both (with almost $40 \%$ of subjects improving by more than $30 \%$ in those measurements), changes that were significant despite high gait variability in response (as shown by large standard deviations). We speculate that the hardware required for the delivery of augmented sensory cues hindered the within-visit device-on response (by partially obstructing the visual field, adding discomfort with the belt sensor, etc.), which explains the greater benefits seen immediately after device removal compared with when the device was in use. This effect is expected to diminish with regular training and use of the device. Furthermore, improvement in the FOGQ, an instrument highly correlated with other measures of quality of life in PD [17], suggests that the changes in gait were clinically signifi- cant. Patients with more advanced disease showed greater benefit after using the device, as greater baseline disability would provide the opportunity for a proportionally larger magnitude of benefit whereas small baseline disability makes it harder for the intervention to show any beneficial effects. The reduction in at-home freezing severity noted in the FOGQ, although small in magnitude, is particularly relevant because of the pharmacoresistance of freezing and its association with an increased rate of falls [18]. A possible mechanism for the reduction in freezing, we speculate, is the generation of cortical reorganization or neuroplasticity, which may alter or circumvent the neuronal pathways responsible for this phenomenon. Finally, the improvement in velocity documented here was dependent on increases in stride length, at the relative expense of cadence. The lack of change in cadence is a reflection of the decrease in step frequency seen when slow to medium walking velocities are replaced with higher walking velocities resulting from increased stride length [19].

This is the first study examining the effects of a virtual (or augmented) reality device for at-home use aimed at improving gait in patients with PD. A prior randomized crossover trial of an external cue training program provided at home by physiotherapists demonstrated small but significant improvements in the Posture and Gait Scale score, a composite of related UPDRS items (13-15 and 29-30) [7]. While that study was the first controlled trial that used a cueing device at home, it relied on an open-loop system with cueing parameters that needed separate adjustments to increase stride length and avoid festination and a training program led by a rehabilitation specialist. These features hinder its feasibility for wider application. Further, 67 percent of study subjects preferred auditory cueing over visual cueing (light flashes delivered through a light-emitting diode attached to a pair of glasses). Auditory cues may be less effective than visual cues [20].

The rehabilitation value of visual cueing, among other sensory feedback, was entered into the literature by Martin [9]. He suggested that the placement of visual cues perpendicular to the direction of gait spaced one step length apart were most effective in improving gait in patients with PD. Many single-session studies have confirmed the benefit of visual cues (high-contrast transverse floor lines) [21-24]. However these open-loop feedback systems may not have long-term effects [25] unless a dedicated physical training program is established, as 
demonstrated in a patient trained to walk on floor cues reaching 120 percent of the uncued stride length over a 1-month period [26]. Closed-loop feedback systems do not require training program monitoring and may lead to long-term motor skills learning and enhancement of adaptive cerebral plasticity, particularly with the use of visual information on which the generation of motor plans is dependent in patients with PD [27]. While an earlier study found closed-loop systems to be safer for patients [3], selected patients may benefit from open-loop systems as well [5]. It is important to emphasize that adherence to a daily training program (minimum of 30 minutes of use twice per day) was critical to realizing the value of the closed-loop feedback system evaluated in this study, because immediate effects were not significant. The training effort needed at the initial visit was minimal, and patients encountered little difficulty in using this device at home without further guidance.

\section{Limitations of this Study}

We must point out several limitations in our study. First, the absence of a control group was unavoidable given the device's lack of a "neutral mode" (e.g., white noise and nontiled visual feedback), which could have served as an "active" placebo. Also due to safety reasons, we could not ethically justify a placebo arm in this athome, unsupervised gait rehabilitation type of intervention. As such, patients were unblinded to the intervention, and a potential order effect from the study conditions could have been present. The main outcome measures relied on electronic-walkway rather than examiner-generated data to compensate for these methodological limitations. The lack of within-visit improvement in our patients, possibly related to subjects' cautious approach to the study hardware, argues against a placebo response although does not rule out physiological test-retest variability in the gait of patients with PD. Second, although we requested and verbally confirmed compliance with the requested two 30-minute training sessions per day, we did not individually monitor device use at home. This precluded assessment of a dose-response effect and subjects' preference for isolated visual or auditory feedback. The device was not uniformly accepted, and in two of the subjects, gait actually worsened while they were using it. In a similar vein, our small study did not have enough power to identify clinical variables associated with improvement versus deterioration. Similarly, the study design precluded our ability to ascertain the effect of single versus dual sensory cueing on gait, since subjects were instructed to use both visual and auditory cueing (though personal preference forced some to rely on only one or none). These issues, along with the decrease in gait performance with device use at baseline that was contrary to what was found in other device-based interventions, may cause concern regarding safe use of the device in a therapist-free intervention, compromised compliance, and increased variability, rendering the interpretation of our results preliminary. The high variability of effect, as well as its delayed occurrence, can very likely be attributed to the learning process associated with training with and using the device, which is highly variable and depends on patient's age and cognitive and mental capabilities. Finally, the off-state UPDRS scores were not matched at the two off-state assessments. The motor improvement at visit 2 was partly driven by a reduction in the axial scores, with which gait velocity has been found to correlate [28], and was not explained by changes in medications or differences at the time of testing. This overall motor improvement may have acted as a confounder in the relationship between sensory cues and gait improvement. Prior studies have also shown nonpharmacological improvement in overall motor performance, such as home-based rehabilitation programs [29], and with other sensory stimulation, such as music [30]. This is also highlighted by the discrepancy often found during routine clinic assessments whereby patients perform better (e.g., less freezing) than what they report to experience in their homes, possibly because of the heightened attention and expectation during the increased scrutiny of clinic visits. Indeed, the testing sessions at the office likely inflated our subjects' "true" off-state motor function and raised the functional baseline against which postintervention gait parameters were compared.

\section{Future Research}

Gait impairment is a major source of disability in patients with PD for which medical and surgical strategies offer relatively little. The noninvasive and effective sensory feedback device used here may prove to be a reasonable therapeutic option for some of these patients. Further studies should ascertain the features associated with responsiveness and lack thereof, the additive effect of combined sensory cueing versus visual- and auditoryonly cueing, the length of the residual benefit after training (to determine the appropriate interval that may suffice to sustain a favorable gait performance in between uses of 
device), the effect of this intervention on the less common but more intractable "on"-state freezing [31], and the benefit, if any, among postsubthalamic deep brain stimulation patients with treatment- and stimulationrefractory freezing of gait.

\section{CONCLUSIONS}

Devices using closed-loop sensory feedback, through an at-home training program, seem effective and desirable nonpharmacologic interventions to improve walking in patients with PD. The device helped people with PD by improving gait and decreasing freezing, which are expected to have an impact on functional mobility and quality of life. Further research is necessary to quantify duration of the residual benefit and applicability to other clinical situations in patients with PD.

\section{ACKNOWLEDGMENTS}

\author{
Author Contributions: \\ Study concept and design: A. J. Espay, Y. Baram, F. J. Revilla. \\ Acquisition of data: A. J. Espay, M. Gartner, L. Gaines, A. P. Duker. \\ Analysis and interpretation of data: A. J. Espay, A. Kumar Dwivedi, \\ R. Shukla. \\ Drafting of manuscript: A. J. Espay. \\ Critical revision of manuscript for important intellectual content: \\ Y. Baram, R. Shukla. \\ Statistical analysis: A. Kumar Dwivedi, R. Shukla. \\ Obtained funding: A. J. Espay. \\ Administrative, technical, or material support: A. J. Espay, Y. Baram. \\ Study supervision: Y. Baram, F. J. Revilla.
}

Financial Disclosures: Dr. Yoram Baram is the inventor and developer of the augmented reality device. He had no involvement in data collection.

Funding/Support: This material was based on work supported in part by resources of the Davis Phinney Foundation for Parkinson Disease Research.

Institutional Review: All subjects provided informed consent for a protocol approved by the University of Cincinnati Institutional Review Board.

Participant Follow-Up: The authors plan to share a copy of this article with each of the study participants.

\section{REFERENCES}

1. Giladi N, McDermott MP, Fahn S, Przedborski S, Jankovic J, Stern M, Tanner C; Parkinson Study Group. Freezing of gait in PD: Prospective assessment in the DATATOP cohort. Neurology. 2001;56(12):1712-21. [PMID: 11425939]

2. Lim I, Van Wegen E, De Goede C, Deutekom M, Nieuwboer A, Willems A, Jones D, Rochester L, Kwakkel G. Effects of external rhythmical cueing on gait in patients with Parkinson's disease: A systematic review. Clin Rehabil. 2005;19(7):695-713. [PMID: 16250189]

DOI:10.1191/0269215505cr906oa

3. Baram Y, Aharon-Peretz J, Simionovici Y, Ron L. Walking on virtual tiles. Neural Proc Lett. 2002;16(3):227-33. DOI:10.1023/A:1021778608344

4. Ferrarin M, Rabuffetti M, Tettamanti M, Pignatti R, Mauro A, Albani G. Effect of optical flow versus attentional strategy on gait in Parkinson's Disease: A study with a portable optical stimulating device. J Neuroeng Rehabil. 2008;5:3. [PMID: 18205903] DOI:10.1186/1743-0003-5-3

5. Protero J. The treatment of akinesia using virtual images [master's thesis]. [Seattle (WA)]: University of Washington, College of Engineering; 1993.

6. Thaut MH, McIntosh GC, Rice RR, Miller RA, Rathbun J, Brault JM. Rhythmic auditory stimulation in gait training for Parkinson's disease patients. Mov Disord. 1996;11(2): 193-200. [PMID: 8684391]

DOI:10.1002/mds.870110213

7. Nieuwboer A, Kwakkel G, Rochester L, Jones D, Van Wegen E, Willems AM, Chavret F, Hetherington V, Baker $\mathrm{K}$, Lim I. Cueing training in the home improves gaitrelated mobility in Parkinson's disease: The RESCUE trial. J Neurol Neurosurg Psychiatry. 2007;78(2):134-40.

[PMID: 17229744] DOI:10.1136/jnnp.200X.097923

Erratum in: J Neurol Neurosurg Psychiatry. 2010;81(1):126.

8. Athans M, Falb PL. Optimal control: An introduction to the theory and its applications. New York (NY): McGraw-Hill; 1966.

9. Martin JP. Locomotion and the basal ganglia. In: Martin JP, editor. The basal ganglia and posture. London (UK): Pitman Medical; 1967. p. 20-35.

10. Baram Y. Walking on tiles. Neural Proc Lett. 1999;10(2): 81-87. DOI:10.1023/A:1018713516431

11. Rougier P. Influence of visual feedback on successive control mechanisms in upright quiet stance in humans assessed by fractional Brownian motion modelling. Neurosci Lett. 1999;266(3):157-60. [PMID: 10465697] DOI:10.1016/S0304-3940(99)00272-4

12. Baram Y, Miller A. Virtual reality cues for improvement of gait in patients with multiple sclerosis. Neurology. 2006; 66(2):178-81. [PMID: 16434649] DOI:10.1212/01.wnl.0000194255.82542.6b

13. Webster KE, Wittwer JE, Feller JA. Validity of the GAITRite walkway system for the measurement of averaged 
and individual step parameters of gait. Gait Posture. 2005;22(4):317-21. [PMID: 16274913]

DOI:10.1016/j.gaitpost.2004.10.005

14. Fahn S, Elton RL. Unified Parkinson's Disease Rating Scale. In: Fahn S, editor. Recent developments in Parkinson's disease. New York (NY): Raven Press; 1987. p. 153-63.

15. Giladi N, Shabtai H, Simon ES, Biran S, Tal J, Korczyn AD. Construction of freezing of gait questionnaire for patients with Parkinsonism. Parkinsonism Relat Disord. 2000;6(3):165-70. [PMID: 10817956] DOI:10.1016/S1353-8020(99)00062-0

16. Espay AJ, Li JY, Johnston L, Chen R, Lang AE. Mirror movements in parkinsonism: Evaluation of a new clinical sign. J Neurol Neurosurg Psychiatry. 2005;76(10):1355-58. [PMID: 16170075$]$

DOI:10.1136/jnnp.2005.062950

17. Moore O, Peretz C, Giladi N. Freezing of gait affects quality of life of peoples with Parkinson's disease beyond its relationships with mobility and gait. Mov Disord. 2007; 22(15): 2192-95. [PMID: 17712856]

DOI:10.1002/mds.21659

18. Gray P, Hildebrand K. Fall risk factors in Parkinson's disease. J Neurosci Nurs. 2000;32(4):222-28.

[PMID: 10994536]

19. Morris ME, Iansek R, Matyas TA, Summers JJ. Ability to modulate walking cadence remains intact in Parkinson's disease. J Neurol Neurosurg Psychiatry. 1994;57(12):1532-34. [PMID: 7798986]

DOI:10.1136/jnnp.57.12.1532

20. Cubo E, Leurgans S, Goetz CG. Short-term and practice effects of metronome pacing in Parkinson's disease patients with gait freezing while in the 'on' state: Randomized single blind evaluation. Parkinsonism Relat Disord. 2004;10(8): 507-10. [PMID: 15542012] DOI:10.1016/j.parkreldis.2004.05.001

21. Morris ME, Iansek R, Matyas TA, Summers JJ. Stride length regulation in Parkinson's disease. Normalization strategies and underlying mechanisms. Brain. 1996;119(Pt 2): 551-68. [PMID: 8800948]

DOI:10.1093/brain/119.2.551

22. Lewis GN, Byblow WD, Walt SE. Stride length regulation in Parkinson's disease: The use of extrinsic, visual cues. Brain. 2000;123(Pt 10):2077-90. [PMID: 11004125] DOI:10.1093/brain/123.10.2077

23. Jiang Y, Norman KE. Effects of visual and auditory cues on gait initiation in people with Parkinson's disease. Clin Rehabil. 2006;20(1):36-45. [PMID: 16502748] DOI:10.1191/0269215506cr925oa

24. Van Wegen E, De Goede C, Lim I, Rietberg M, Nieuwboer A, Willems A, Jones D, Rochester L, Hetherington V, Berendse H, Zijlmans J, Wolters E, Kwakkel G. The effect of rhythmic somatosensory cueing on gait in patients with
Parkinson's disease. J Neurol Sci. 2006;248(1-2):210-14. [PMID: 16780887]

DOI:10.1016/j.jns.2006.05.034

25. Rochester L, Nieuwboer A, Baker K, Hetherington V, Willems AM, Chavret F, Kwakkel G, Van Wegen E, Lim I, Jones D. The attentional cost of external rhythmical cues and their impact on gait in Parkinson's disease: Effect of cue modality and task complexity. J Neural Transm. 2007; 114(10):1243-48. [PMID: 17598068] DOI:10.1007/s00702-007-0756-y

26. Sidaway B, Anderson J, Danielson G, Martin L, Smith G. Effects of long-term gait training using visual cues in an individual with Parkinson disease. Phys Ther. 2006;86(2): 186-94. [PMID: 16445332]

27. Helmich RC, De Lange FP, Bloem BR, Toni I. Cerebral compensation during motor imagery in Parkinson's disease. Neuropsychologia. 2007;45(10):2201-15. [PMID: 17448507$]$ DOI:10.1016/j.neuropsychologia.2007.02.024

28. Vieregge P, Stolze H, Klein C, Heberlein I. Gait quantification in Parkinson's disease-Locomotor disability and correlation to clinical rating scales. J Neural Transm. 1997; 104(2-3):237-48. [PMID: 9203085]

DOI:10.1007/BF01273184

29. Caglar AT, Gurses HN, Mutluay FK, Kiziltan G. Effects of home exercises on motor performance in patients with Parkinson's disease. Clin Rehabil. 2005;19(8):870-77.

[PMID: 16323386]

DOI:10.1191/0269215505cr924oa

30. Pacchetti C, Aglieri R, Mancini F, Martignoni E, Nappi G. Active music therapy and Parkinson's disease: Methods. Funct Neurol. 1998;13(1):57-67. [PMID: 9584875]

31. Kompoliti K, Goetz CG, Leurgans S, Morrissey M, Siegel IM. “On” freezing in Parkinson's disease: Resistance to visual cue walking devices. Mov Disord. 2000;15(2):309-12.

[PMID: 10752582]

DOI:10.1002/1531-8257(200003)15:2<309::AIDMDS1016>3.0.CO;2-P

Submitted for publication October 14, 2009. Accepted in revised form March 30, 2010.

This article and all supplementary material should be cited as follows:

Espay AJ, Baram Y, Kumar Dwivedi A, Shukla R, Gartner M, Gaines L, Duker AP, Revilla FJ. At-home training with closed-loop augmented-reality cueing device for improving gait in patients with Parkinson disease. J Rehabil Res Dev. 2010;47(6):573-82

DOI:10.1682/JRRD.2009.10.0165 
